\title{
Counterterms vs. dualities
}

\section{Guillaume Bossard ${ }^{a}$ and Hermann Nicolai ${ }^{b}$}

${ }^{a}$ Centre de Physique Théorique, Ecole Polytechnique, CNRS, 91128 Palaiseau Cedex, France

${ }^{b}$ AEI, Max-Planck-Institut für Gravitationsphysik, Am Mühlenberg 1, D-14476 Potsdam, Germany

E-mail: bossard@cpht.polytechnique.fr, Hermann.Nicolai@aei.mpg.de

ABSTRACT: We investigate and clarify the mutual compatibility of the higher order corrections arising in supergravity and string theory effective actions and the non-linear duality symmetries of these theories. Starting from a conventional tree level action leading to duality invariant equations of motion, we show how to accommodate duality invariant counterterms given as functionals of both electric and magnetic fields in a perturbative expansion, and to deduce from them a non-polynomial bona fide action satisfying the Gaillard-Zumino (NGZ) constraint. There exists a corresponding consistency constraint in the non-covariant Henneaux-Teitelboim formalism which ensures that one can always restore diffeomorphism invariance by perturbatively solving this functional identity. We illustrate how this procedure works for the $R^{2} \nabla F \nabla F$ and $F^{4}$ counterterms in Maxwell theory.

KeYwords: Supersymmetry and Duality, Extended Supersymmetry, Supergravity Models, Anomalies in Field and String Theories

ARXIV EPRINT: 1105.1273 


\section{Contents}

1 Introduction 1

2 Deformed twisted selfduality constraint $\quad 3$

$\begin{array}{lll}3 & \text { Non-covariant formulation with manifest duality } & 6\end{array}$

4 Maxwell theory, once again $\quad 10$

5 Conclusions 13

$\begin{array}{ll}\text { A Deforming the NGZ identity } & 13\end{array}$

\section{Introduction}

Consider Einstein theory coupled to scalar fields parametrising a symmetric space $G / K(G)$ (where $K(G)$ is the maximal compact subgroup of $G$ ), and $n$ abelian vector fields such that $G \subset \operatorname{Sp}(2 n, \mathbb{R})$ acts linearly on them and their magnetic duals. This setup is typical for the bosonic sector of various (ungauged) extended supergravity theories, and in particular for the maximally extended $\mathcal{N}=8$ supergravity with duality group $G=E_{7(7)} \subset \operatorname{Sp}(56, \mathbb{R})[1]$. The purpose of this letter is to discuss the consistency of the action of this duality group, when higher order local corrections to the tree level action (of the type appearing in the string theory effective action or as counterterms in extended supergravities) are included. Accordingly, we will consider $n$ 'electric' vector fields $A_{\mu}^{m}$ together with their 'magnetic' duals $A_{\mu}^{\bar{m}}$, combining them into a $2 n$-plet of vectors $A_{\mu}^{m}$ with $2 n$ associated field strengths $F_{\mu \nu}^{m}$, viz.

$$
A_{\mu}^{m} \equiv\left(A_{\mu}^{m}, A_{\mu}^{\bar{m}}\right) \quad \Leftrightarrow \quad F_{\mu \nu}^{m} \equiv\left(F_{\mu \nu}^{m}, F_{\mu \nu}^{\bar{m}}\right)
$$

Note that the $n$ magnetic duals $A_{\mu}^{\bar{m}}$ are only defined on shell, as non-local functionals of the other fields of the theory. ${ }^{1}$ Classically, this redundancy is reflected in the so-called twisted selfduality constraint [1] for the $2 n$ field strengths $F_{\mu \nu}^{m}$

$$
F_{\mu \nu}^{m}=-\frac{1}{2 \sqrt{-g}} \varepsilon_{\mu \nu}^{\sigma \rho} J^{m}{ }_{n} F_{\sigma \rho}^{n},
$$

which simultaneously halves the number of degrees of freedom and puts the theory onshell, in such a way that the Bianchi identities for the electric vectors imply the equations

\footnotetext{
${ }^{1}$ In the literature [2-4] the magnetic field strengths are often denoted by the letter $G_{\mu \underline{\nu}}$, so the relation with our notation (which follows [5]) is established by making the identification $\left(F_{\mu \nu}^{m}, F_{\mu \nu}^{\bar{m}}\right) \equiv\left(F_{\mu \nu}^{m}, G_{\mu \nu}^{m}\right)$. Because the extension of our arguments to fermions is straightforward, we will not consider fermions in this paper, but see e.g. [6].
} 
of motion for the magnetic vectors, and vice versa. Here, $J^{m}{ }_{n}$ is a 'complex structure' built from the $\operatorname{Sp}(2 n, \mathbb{R})$ invariant symplectic form $\Omega^{m n}$ and the scalar field dependent symmetric metric $G_{m n} \in G$

$$
J_{n}^{m} \equiv \Omega^{m p} G_{p n} \quad \Rightarrow \quad J^{m}{ }_{p}{ }_{n}=-\delta_{n}^{m}
$$

The indices $(m, \bar{m})$ correspond to the decomposition (1.1) of the $2 n$ vectors in a Darboux basis such that the symplectic form splits as

$$
\Omega_{m n}=\Omega_{\bar{m} \bar{n}}=0, \quad \Omega_{m \bar{n}}=-\Omega_{\bar{n} m}=\delta_{m \bar{n}},
$$

Defining $H^{\bar{m} \bar{n}}$ as the inverse of $G_{\bar{m} \bar{n}}$ one directly obtains from (1.2)

$$
F_{\mu \nu}^{\bar{m}}=H^{\bar{m} \bar{n}}\left(\delta_{\bar{n} m} \frac{1}{2 \sqrt{-g}} \varepsilon_{\mu \nu}^{\sigma \rho} F_{\sigma \rho}^{m}-G_{\bar{n} m} F_{\mu \nu}^{m}\right) .
$$

The classical action is then re-obtained by solving the equation

$$
F_{\mu \nu}^{\bar{m}}=-\delta^{\bar{m} m} \frac{1}{-g} \varepsilon_{\mu \nu \sigma \rho} \frac{\delta S}{\delta F_{\sigma \rho}^{m}}
$$

with the result

$$
S \equiv S^{(0)}=-\frac{1}{4} \int d^{4} x\left(\sqrt{-g} H^{\bar{m} \bar{n}} \delta_{\bar{m} m} \delta_{\bar{n} n} F^{m \mu \nu} F_{\mu \nu}^{n}+\frac{1}{2} \varepsilon^{\mu \nu \sigma \rho} H^{\bar{m} \bar{n}} G_{\bar{n} n} \delta_{\bar{m} m} F_{\mu \nu}^{m} F_{\sigma \rho}^{n}\right) .
$$

As required, the tree level action $S^{(0)}\left[F_{\mu \nu}^{m}\right]$ depends only on the electric vector potentials. For more general actions $S$ depending on the electric vector fields, the basic relations (1.6) remain the same, and are usually referred to as constitutive relations [3]. As shown in [2,3] it is a general feature that the action itself is not duality invariant, but varies as

$$
\delta^{\mathfrak{g}} S\left[F_{\mu \nu}^{m}\right]=\frac{1}{8} \int d^{4} x\left(\varepsilon^{\mu \nu \sigma \rho} X^{\bar{m}}{ }_{n} \delta_{\bar{m} m} F_{\mu \nu}^{m} F_{\sigma \rho}^{n}-\frac{4}{-g} \varepsilon_{\mu \nu \sigma \rho} X^{m}{ }_{\bar{n}} \delta^{\bar{n} n} \frac{\delta S}{\delta F_{\mu \nu}^{m}} \frac{\delta S}{\delta F_{\sigma \rho}^{n}}\right)
$$

under the duality transformations

$$
\delta^{\mathfrak{g}} G^{m n}=X^{m}{ }_{p} G^{p n}+X^{n}{ }_{p} G^{m p}, \quad \delta^{\mathfrak{g}} F_{\mu \nu}^{m}=X^{m}{ }_{n} F_{\mu \nu}^{n} .
$$

We have omitted the superscript ${ }^{(0)}$ in (1.8) because, as shown in $[2,3]$, the equation (1.8) is the consistency condition for any action $S$ with associated duality invariant equations of motion. ${ }^{2}$ In particular, it must also hold for actions including non-linear deformations or higher order corrections, so that the duality transformations preserve the constitutive relations (1.6).

Suppose now that we are given a classical action $S^{(0)}$ satisfying these requirements, such as for instance the tree level action of $\mathcal{N}=8$ supergravity, whose vector part is just given by (1.7) (for $G=E_{7(7)}$ ). In perturbation theory, this action will be modified by higher

\footnotetext{
${ }^{2}$ Note that (1.8) is required for the duality transformations to make sense on the fields, and this is also valid when they are not symmetries of the equations of motion, but nevertheless admit a representation on the fields satisfying the equations of motion.
} 
order counterterms and corrections whose compatibility with duality transformations and with (1.8) is not immediately obvious. The higher order corrections to the action are only defined modulo the equations of motion of the classical action $S^{(0)}$. They are generally given as functionals of the $2 n$ electric and magnetic vectors $A_{\mu}^{m}$, that is, in the form $\mathcal{I}^{(1)}=\mathcal{I}^{(1)}\left[F_{\mu \nu}^{m}, F_{\mu \nu}^{\bar{m}}\right] .{ }^{3}$ In particular, the higher order counterterms in $\mathcal{N}=8$ supergravity appear generically in this manifestly covariant form with respect to the duality group in terms of vector fields transforming in the linear 56 representation of $E_{7(7)}$ [7-9]. When trying to express the original action together with the corrections as an actual new action functional of the electric field strengths only, we are thus faced with the question what expression to substitute for the magnetic field strengths $F_{\mu \nu}^{\bar{m}}$ : after all, these will be given by non-linear and possibly non-local functionals of the electric vector fields (as well as the other fields) whose form is determined precisely by the new corrected action we are looking for. A naive guess might be to substitute the tree level solution (1.5), but one quickly sees that this ansatz solves the consistency condition (1.8) only to first order in perturbation theory, and fails at higher orders. In other words, it could a priori appear that the corrected action functional gives rise to inconsistencies with the action of the duality transformations $(1.9)[4,10]$.

\section{Deformed twisted selfduality constraint}

To find the right action one must therefore adopt a different strategy, taking a deformed version of the twisted selfduality constraint as the starting point. Namely, given a manifestly duality covariant counterterm correction $\mathcal{I}^{(1)}$ depending on the $2 n$ field strengths $F_{\mu \nu}^{m}$ and their derivatives, we propose to replace (1.2) by the deformed twisted selfduality constraint

$$
F_{\mu \nu}^{m}-\frac{2}{\sqrt{-g}} G^{m n} g_{\mu \sigma} g_{\nu \rho} \frac{\delta \mathcal{I}^{(1)}}{\delta F_{\sigma \rho}^{n}}=-\frac{1}{2 \sqrt{-g}} \varepsilon_{\mu \nu}^{\sigma \rho} J_{n}^{m}\left(F_{\mu \nu}^{m}-\frac{2}{\sqrt{-g}} G^{m n} g_{\sigma \tau} g_{\rho \omega} \frac{\delta \mathcal{I}^{(1)}}{\delta F_{\tau \omega}^{n}}\right)
$$

or, equivalently,

$$
F_{\mu \nu}^{m}+\frac{1}{2 \sqrt{-g}} \varepsilon_{\mu \nu}^{\sigma \rho} J_{n}^{m} F_{\sigma \rho}^{n}=\frac{2}{\sqrt{-g}} G^{m n} g_{\mu \sigma} g_{\nu \rho} \frac{\delta \mathcal{I}^{(1)}}{\delta F_{\sigma \rho}^{n}}+\Omega^{m n} \frac{1}{-2 g} \varepsilon_{\mu \nu \sigma \rho} \frac{\delta \mathcal{I}^{(1)}}{\delta F_{\sigma \rho}^{n}} .
$$

This equation is manifestly duality invariant if $\mathcal{I}^{(1)}$ is a duality invariant functional. At the same time it achieves the required halving of the number of physical degrees of freedom and imposes the (deformed) equations of motion. To reconstruct a bona fide action depending only on the physical fields (and only the electric vector fields, in particular) and satisfying all consistency requirements, we now have two options.

- We first solve (2.1) for the magnetic field strengths $F_{\mu \nu}^{\bar{m}}$ in function of the electric field strengths $F_{\mu \nu}^{m}$ and their derivatives (as well as all other fields) as a formal power series. With the resulting expression for $F_{\mu \nu}^{\bar{m}}$ as a functional of the physical fields, we then solve (1.6) in a second step to obtain the full corrected action functional in terms

\footnotetext{
${ }^{3}$ For clarity of notation, we will use the letter $S$ only for 'true' actions defined off shell, whereas $\mathcal{I}$ denotes a general functional of both electric and magnetic fields.
} 
of the electric vectors only. This procedure manifestly preserves four-dimensional space-time covariance.

- Alternatively, we can solve (2.1) for the time components $\left(F_{0 \mathrm{i}}^{m}, F_{0 \mathrm{i}}^{\bar{m}}\right)$, and again reconstruct the requisite action in a second step. The resulting action depends on the spatial electric and magnetic vector components $\left(A_{\mathrm{i}}^{m}, A_{\mathrm{i}}^{\bar{m}}\right)$, and therefore breaks manifest space-time covariance. Nevertheless, we will see that there is a consistency condition that guarantees full space-time covariance on-shell.

Due to the non-linear dependence of $\mathcal{I}^{(1)}$ on the magnetic field strengths $F_{\mu \nu}^{\bar{m}}$ and possibly their derivatives, the resulting corrected action will include terms of arbitrarily high order for any kind of counterterm correction, and this will be true in both approaches. In other words, the 'initial' counterterm $\mathcal{I}^{(1)}$, which is usually polynomial in the field strengths and their derivatives, must be supplemented by an infinite string of higher order terms. This completion of the 'initial' counterterm action will thus be non-polynomial, and also nonlocal if the initial counterterm depends on derivatives. ${ }^{4}$ Yet, it will satisfy the consistency condition (1.8).

Let us illustrate these claims with a simple example from Maxwell theory, adopting the space-time covariant approach. For this purpose we combine the electric vector $A_{\mu}^{1}$ with its magnetic dual $A_{\mu}^{2} \equiv A_{\mu}^{\overline{1}}$ into a complex vector potential $A_{\mu} \equiv A_{\mu}^{1}+i A_{\mu}^{2}$, with corresponding complex field strength

$$
F_{\mu \nu}=F_{\mu \nu}^{1}+i F_{\mu \nu}^{2}
$$

Electromagnetic U(1) duality then acts on these fields simply as a global phase rotation. It is furthermore easy to see that the original (free) Maxwell equations for $A_{\mu}^{1}$ are recovered from the twisted selfduality constraint

$$
F_{\mu \nu}^{-}=0,
$$

where we define the complex selfdual and anti-selfdual field strengths as

$$
F_{\mu \nu}^{ \pm}:=\frac{1}{2} F_{\mu \nu} \pm \frac{i}{4 \sqrt{-g}} \varepsilon_{\mu \nu}^{\sigma \rho} F_{\sigma \rho} .
$$

As an example of a non-trivial deformation let us consider the $\mathrm{U}(1)$ duality invariant expression

$$
\mathcal{I}^{(1)}=-\frac{1}{4} \int d^{4} x \sqrt{-g} g^{\rho \kappa} T^{\mu \nu \sigma \lambda} \nabla_{\mu} \bar{F}_{\sigma \rho} \nabla_{\nu} F_{\lambda \kappa},
$$

where $T^{\mu \nu \sigma \rho}$ is the Bel-Robinson tensor

$$
T^{\mu \nu \sigma \rho} \equiv C^{\mu \kappa \sigma \lambda} C^{\nu}{ }_{\kappa}{ }^{\rho}{ }_{\lambda}-\frac{3}{2} g^{\mu[\nu} C^{\kappa \lambda] \sigma \vartheta} C_{\kappa \lambda}{ }^{\rho} \vartheta,
$$

with the Weyl tensor $C_{\mu \nu \sigma \rho}$. The Bel-Robinson tensor is fully symmetric and traceless in its four indices and is conserved modulo the vacuum equations of motion. With the

\footnotetext{
${ }^{4}$ Note, however, that it will nevertheless remain local in a perturbative sense, i.e. involve only a finite number of derivatives at any given order in the coupling constant.
} 
above notation, the deformed twisted selfduality constraint for our Maxwell example takes the form

$$
F_{\mu \nu}^{-}+\nabla_{\sigma} T_{[\mu}^{\sigma \rho \lambda} \nabla_{\rho} F_{\nu] \lambda}^{+}=0
$$

Observe that the second term in (2.8) is complex anti-selfdual in the indices $[\mu \nu]$, as it should be, because the Bel-Robinson tensor is symmetric traceless and the torsion-free covariant derivatives preserve complex (anti)selfduality.

Let us now construct a manifestly diffeomorphism covariant Lagrangian for the deformed equations of motion in terms of the real Maxwell field strengths $F_{\mu \nu}^{1}$ only, following the above procedure. To this aim we define the differential operator

$$
(\Delta(f))_{\mu \nu} \equiv \Delta_{\mu \nu}^{\rho \sigma} f_{\rho \sigma}:=\nabla_{\kappa} T_{[\mu}^{\kappa \lambda[\sigma} \nabla_{\lambda} \delta_{\nu]}^{\rho]} f_{\rho \sigma} .
$$

acting on two-forms $f_{\mu \nu}$. This operator is self-adjoint and satisfies

$$
\frac{1}{2 \sqrt{-g}} \varepsilon_{\mu \nu}^{\kappa \lambda} \Delta_{\kappa \lambda}^{\sigma \rho}=-\Delta_{\mu \nu}{ }^{\kappa \lambda} \frac{1}{2 \sqrt{-g}} \varepsilon_{\kappa \lambda}{ }^{\sigma \rho},
$$

thus converting selfdual into anti-selfdual tensors, and vice versa (this accounts for the opposite duality phases on $F_{\nu \lambda}^{+}$in (2.8)). Decomposing (2.8) one obtains

$$
\left(\delta_{\mu \nu}^{\sigma \rho}+\Delta_{\mu \nu}^{\sigma \rho}\right) F_{\sigma \rho}^{2}=\left(\delta_{\mu \nu}^{\sigma \rho}-\Delta_{\mu \nu}^{\sigma \rho}\right) \frac{1}{2} \varepsilon_{\sigma \rho}^{\kappa \lambda} F_{\kappa \lambda}^{1},
$$

and inverting the operator on the left-hand side we get

$$
F_{\mu \nu}^{2}=\frac{1}{2} \varepsilon_{\mu \nu} \kappa \lambda\left(\delta_{\kappa \lambda}^{\sigma \rho}+2 \sum_{n \geq 1}\left(\Delta^{n}\right)_{\kappa \lambda}^{\sigma \rho}\right) F_{\sigma \rho}^{1},
$$

where $\Delta^{n}$ is the $n^{\text {th }}$ power of $\Delta$. This equation, in turn, simply follows as the EulerLagrange equation of the action

$$
S=-\int d^{4} x \sqrt{-g}\left(\frac{1}{4} F^{1 \mu \nu} F_{\mu \nu}^{1}+\frac{1}{2} \sum_{n=1}^{\infty} F^{1 \mu \nu}\left(\Delta^{n}\right)_{\mu \nu}{ }^{\sigma \rho} F_{\sigma \rho}^{1}\right) .
$$

The completion of the 'initial' Bel-Robinson counterterm $\mathcal{I}^{(1)}$ from (2.6) is thus nonpolynomial and also non-local (depending on arbitrarily high powers of the derivative operator $\nabla_{\mu}$ ). To check the consistency condition (1.8) we first observe that

$$
\begin{aligned}
\int d^{4} x \frac{2}{-g} \varepsilon_{\mu \nu \sigma \rho} \frac{\delta S}{\delta F_{\mu \nu}^{1}} \frac{\delta S}{\delta F_{\sigma \rho}^{1}} & =\int d^{4} x \frac{1}{2} \varepsilon^{\mu \nu \sigma \rho}\left(\delta_{\mu \nu}^{\kappa \lambda}+2 \sum_{n \geq 1} \Delta_{\mu \nu}^{n \kappa \lambda}\right) F_{\kappa \lambda}^{1}\left(\delta_{\sigma \rho}^{\theta \tau}+2 \sum_{n \geq 1} \Delta_{\sigma \rho}^{n \theta \tau}\right) F_{\theta \tau}^{1} \\
& =\int d^{4} x \frac{1}{2} \varepsilon^{\mu \nu \sigma \rho} F_{\mu \nu}^{1}\left(\delta_{\sigma \rho}^{\kappa \lambda}+2 \sum_{n \geq 1}(-\Delta)_{\sigma \rho}^{n \kappa \lambda}\right)\left(\delta_{\kappa \lambda}^{\theta \tau}+2 \sum_{n \geq 1} \Delta_{\kappa \lambda}^{n \theta \tau}\right) F_{\theta \tau}^{1} \\
& =\int d^{4} x \frac{1}{2} \varepsilon^{\mu \nu \sigma \rho} F_{\mu \nu}^{1} F_{\sigma \rho}^{1} .
\end{aligned}
$$

Because $X_{2}^{1}=-X_{1}^{2}$ for a $\mathrm{U}(1)$ duality rotation, this means that the two terms on the right-hand side of (1.8) are the same, yielding twice the right-hand side of (2.14). Now 
using $\delta^{\mathfrak{u}(1)} F_{\mu \nu}^{1}=X^{1}{ }_{2} F_{\mu \nu}^{2}$ together with the constitutive relations (1.6), it is straightforward to see that (1.8) is indeed satisfied for the completed action (2.13).

The counterterm (2.6) is actually a simplified version of a typical term appearing in the supersymmetric completion of the $R^{4}$ counterterm arising at three loops in $\mathcal{N}=8$ supergravity [11], ${ }^{5}$ where it is proportional to (using $\operatorname{SL}(2, \mathbb{C})$ spinor notation)

$$
C^{\alpha \beta \gamma \delta} C^{\dot{\alpha} \dot{\beta} \dot{\gamma} \dot{\delta}} \nabla_{\alpha \dot{\delta}} F_{\beta \gamma}^{i j} \nabla_{\delta \dot{\alpha}} \bar{F}_{\dot{\beta} \dot{\gamma} i j}
$$

with the $\mathrm{SU}(8)$ field strength $F_{\alpha \beta}^{i j} \equiv \sigma_{\alpha \beta}^{\mu \nu} F_{\mu \nu}^{i j}$ and its complex conjugate $\bar{F}_{\dot{\alpha} \dot{\beta} i j}$. It is rather straightforward to generalize the above calculation and to obtain the corresponding piece of the corrected action of $\mathcal{N}=8$ supergravity to all orders. More specifically, with the notation and the conventions of [15] we get

$$
\begin{aligned}
S=-\frac{1}{2} \int d^{4} x \sqrt{-g}( & \frac{1}{2} \operatorname{Re}[2 S-1]^{I J, K L} F^{I J \mu \nu} F_{\mu \nu}^{K L} \\
& +\frac{1}{2 \sqrt{-g}} \varepsilon^{\mu \nu \sigma \rho} \operatorname{Im}[S]^{I J K L} F_{\mu \nu}^{I J} F_{\sigma \rho}^{K L} \\
& \left.+F^{I J \mu \nu} \sum_{n}\left(\Delta^{n}\right)_{\mu \nu}{ }^{\sigma \rho I J, K L} \operatorname{Re}[2 S-1]^{K L, P Q} F_{\sigma \rho}^{P Q}\right)
\end{aligned}
$$

where $F_{\mu \nu}^{I J}$ are the real field strengths associated with the 28 Maxwell vectors of $\mathcal{N}=8$ supergravity. The operator $\Delta^{I J, K L}$ is defined from (2.9) by covariantizing the differential operator $\nabla_{\mu}$ also w.r.t. the local $\mathrm{SU}(8)$ symmetry of $\mathcal{N}=8$ supergravity, such that

$$
\begin{aligned}
\Delta_{\mu \nu}{ }^{\sigma \rho I J, K L}=\operatorname{Re}[ & \left.K^{I J}{ }_{i j} \Delta_{\mu \nu}{ }^{\sigma \rho}\left(u^{i j} K L+v^{i j K L}\right)\right] \\
& +\frac{1}{2 \sqrt{-g}} \varepsilon_{\mu \nu}{ }^{\kappa \lambda} \operatorname{Im}\left[K^{I J}{ }_{i j} \Delta_{\kappa \lambda}{ }^{\sigma \rho}\left(u^{i j} K L+v^{i j K L}\right)\right]
\end{aligned}
$$

with

$$
K^{I J}{ }_{i j}\left(u^{i j}{ }_{K L}+v^{i j K L}\right)=\delta_{K L}^{I J}, \quad S^{I J, K L}=K^{I J}{ }_{i j} u^{i j}{ }_{K L}
$$

To be sure, this argument says nothing about the deformation of local supersymmetry that must also be taken into account when counterterms are added to the original action of $\mathcal{N}=8$ supergravity. To carry out such a computation in full and explicit detail appears beyond reach, but in the following section we will present general arguments (based on the absence of diffeomorphism and local supersymmetry anomalies in four dimensions) that a fully invariant deformation simultaneously compatible with nonlinear supersymmetry and $E_{7(7)}$ exists and can be obtained at least in principle in an order by order calculation.

\section{Non-covariant formulation with manifest duality}

In the foregoing section we showed how to restore the full duality invariance for the corrected equations of motion. However, being on-shell, this formalism is not directly suited

\footnotetext{
${ }^{5}$ Note that the complete supersymmetry invariant is not actually duality invariant $[12,13]$, however its non-perturbative completion arising in string theory is believed to be $E_{7(7)}(\mathbb{Z})$ invariant (see e.g. [14]), and so it is important that it transforms covariantly with respect to $E_{7(7)}$.
} 
for quantisation because we cannot formulate the functional Ward identities for the duality symmetry in that case. For that purpose one must instead make use of a non-covariant formulation developed by Henneaux and Teitelboim [16], and worked out for $\mathcal{N}=8$ supergravity by Hillmann [6] (see also [5]). In that formalism one takes the $2 n$ spatial three-vectors $A_{i}^{m}$ as the fundamental fields, while their time components are only defined on-shell. As a consequence, the action is manifestly duality invariant, but no longer manifestly invariant under space-time diffeomorphisms. At tree level, it takes the form

$$
S_{\mathrm{vec}}^{(0)}=\frac{1}{4} \int d^{4} x\left(\Omega_{m n} \varepsilon^{\mathrm{ijk}}\left(\partial_{0} A_{\mathrm{i}}^{m}+N^{1} F_{\mathrm{il}}^{m}\right) F_{\mathrm{jk}}^{n}-N \sqrt{h} G_{m n} h^{\mathrm{ik}} h^{\mathrm{jl}} F_{\mathrm{ij}}^{m} F_{\mathrm{kl}}^{n}\right) .
$$

It is invariant only with respect to a non-standard realisation of space-time diffeomorphisms (but, of course, still invariant under spatial diffeomorphisms). The equation of motion for the vector fields is

$$
\varepsilon^{\mathrm{ijk}} \partial_{\mathrm{j}} \mathscr{E}_{\mathrm{k}} m=0
$$

with the abbreviation

$$
\mathscr{E}_{\mathrm{i}}^{m} \equiv \partial_{0} A_{\mathrm{i}}^{m}+N^{\mathrm{j}} F_{\mathrm{ij}}^{m}-\frac{N}{2 \sqrt{h}} h_{\mathrm{ij}} \varepsilon^{\mathrm{jkl}} J_{n}^{m} F_{\mathrm{kl}}^{n}
$$

(recall that we neglect fermionic terms). It is invariant with respect to the modified diffeomorphism transformation of the vector field

$$
\delta_{\xi} A_{\dot{i}}^{m} \equiv \xi^{j} F_{j i}^{m}+\xi^{o}\left(\partial_{0} A_{\dot{i}}^{m}-\mathscr{E}_{\dot{i}}^{m}\right)
$$

Although the component $A_{0}^{m}$ of the vector field does not appear in the action, its spatial gradient can be identified from the equations of motion as

$$
\partial_{i} A_{0}^{m}=\mathscr{E}_{i}^{m}
$$

One then expresses the Lorentz field strength $F_{\mu \nu}^{m}$ via $F_{i j}^{m}$ and

$$
F_{0 \mathrm{i}}^{m}=\partial_{0} A_{\dot{\mathrm{i}}}^{m}-\mathscr{E}_{\mathrm{i}}^{m}
$$

With this definition, one checks that the field strength $F_{\mu \nu}^{m}$ transforms indeed as it should with respect to diffeomorphisms modulo the equations of motion

$$
\delta F_{\mu \nu}^{m}=\xi^{\sigma} \partial_{\sigma} F_{\mu \nu}^{m}-2 F_{\sigma[\mu} \partial_{\nu]} \xi^{\sigma}+\xi^{0} \mathscr{E}_{\mu \nu}^{m}
$$

where $\mathscr{E}_{\mu \nu}^{m}$ is the twisted selfdual component of the equations of motion, i.e.

$$
\mathscr{E}_{\mathrm{ij}} m=\Omega^{m n} \varepsilon_{\mathrm{ijk}} \frac{\delta S^{(0)}}{\delta A_{\mathrm{k}}^{n}}, \quad \mathscr{E}_{0 \mathrm{i}}=-N^{\mathrm{j} \mathscr{E}_{\mathrm{ij}} m}+\frac{N}{2 \sqrt{h}} h_{\mathrm{ij}} \varepsilon^{\mathrm{jkl}} J^{m}{ }_{n} \mathscr{E}_{\mathrm{kl}} n
$$

in accord with the (undeformed) twisted selfduality constraint.

Next let us consider some higher order supersymmetric invariant $\mathcal{I}^{(1)}$ defined on-shell as a functional of $F_{\mu \nu}^{m}$ and the other fields of the theory, which is invariant with respect to the 
ordinary action of diffeomorphisms. From this action we directly obtain the corresponding off-shell action $S^{(1)}$ by substituting (3.6) for the time-components $F_{0 \dot{1}}^{m}$, viz.

$$
S^{(1)}\left[F_{i j}^{m}\right] \equiv \mathcal{I}^{(1)}\left[F_{i j}^{m}, F_{0 i}^{m} \equiv \partial_{0} A_{i}^{m}-\mathscr{E}_{\dot{i}}^{m}\right] .
$$

Its variation under a time-like diffeomorphism with parameter $\xi^{0}$ is ${ }^{6}$

$$
\delta S^{(1)}=\int d x^{4} \Omega^{m n} \varepsilon^{\mathrm{ijk}} \xi^{0} \frac{\delta S^{(1)}}{\delta F_{i j}^{m}} \frac{\delta S^{(0)}}{\delta A_{\mathrm{k}}^{n}} .
$$

It follows that, at the same order, the action $S^{(0)}+S^{(1)}$ is invariant with respect to the modified variation

$$
\delta A_{i}^{m}=\xi^{j} F_{j i}^{m}+\xi^{o}\left(\partial_{0} A_{i}^{m}-\mathscr{E}_{i}^{m}-\Omega^{m n} \varepsilon^{i j k} \frac{\delta S^{(1)}}{\delta F_{j k}^{n}}\right) .
$$

At this order this result is precisely the expected one: the diffeormorphism transformation of the vector field agrees with the ordinary transformation modulo the corrected equations of motion. Of course, in order to obtain full agreement and to establish the consistency of the deformed action one must now complete the corrected action by adding higher order terms, just like for the covariant deformed Maxwell action in the previous section. That is, we must determine the full invariant

$$
S=S^{(0)}+S^{(1)}+S^{(2)}+\ldots
$$

with a corresponding all order corrected transformation of the vector fields. The possible obstructions in carrying out this procedure are the solutions of the diffeomorphism WessZumino consistency conditions as functionals of $F_{\mu \nu}^{m}$ and the other fields, identified modulo the equations of motion [17]. Because the action of diffeomorphisms on $F_{\mu \nu}^{m}$ is identical to the conventional one modulo the equations of motion, this cohomology problem is identical to the one of identifying algebraic diffeomorphism anomalies in four dimensions. Consequently, the absence of such anomalies [18, 19] ensures the existence of a completed action $S$ which is invariant with respect to its associated diffeomorphism action.

Similar reasoning permits to argue that the same procedure applies to supersymmetry invariants. The existence of a completed action invariant with respect to supersymmetry relies on the absence of supersymmetry anomalies. However, the argument is less straightforward in that case because the supersymmetry variation of the fermion fields in the Henneaux-Teitelboim formulation coincides with their supersymmetry variation in the conventional (covariant) formulation only modulo the 'integrated' classical equations of motion (3.5). Although proving this is beyond the scope of this paper, we argue that gauge invariance implies that this subtlety does not alter the proof and that the cohomology groups associated to the supersymmetry anomaly are isomorphic in the two formalisms. Equivalently, this would imply the absence of any obstruction in defining the all order supersymmetric action as a formal power series.

\footnotetext{
${ }^{6}$ The covariance under spatial diffeomorphisms (with parameters $\xi^{\dot{1}}$ ) is manifest.
} 
We will now show how to compute the complete action $S$ perturbatively by using the invariance of the action as a first order functional derivative equation. To this aim we consider the action ${ }^{7}$

$$
S=\frac{1}{4} \int d^{4} x\left(\Omega_{m n} \varepsilon^{i j k}\left(\partial_{0} A_{i}^{m}+N^{1} F_{i 1}^{m}\right) F_{j k}^{n}\right)+I\left[F_{i j}^{m}, \nabla_{\mu} F_{i j}^{m}, \ldots\right],
$$

where the functional $I$ depends on the vector fields via the spatial field strengths $F_{i j}^{m}$ and their derivatives (including time derivatives). For any such $I$ the equations of motion of the vector fields still take the form of a spatial divergence

$$
\frac{\delta S}{\delta A_{\mathrm{i}}^{m}}=-\varepsilon^{\mathrm{ijk}} \partial_{\mathrm{j}}\left(\Omega_{m n}\left(\partial_{0} A_{\mathrm{k}}^{n}+N^{\mathrm{l}} F_{\mathrm{kl}}^{n}\right)-\varepsilon_{\mathrm{klh}} \frac{\delta I}{\delta F_{\mathrm{lh}}^{m}}\right)=0,
$$

They are thus equivalent to the first order equation

$$
F_{0 \mathrm{i}}^{m}=-N^{\mathrm{j}} F_{\mathrm{ij}}^{m}-\Omega^{m n} \varepsilon_{\mathrm{ijk}} \frac{\delta I}{\delta F_{\mathrm{jk}}^{n}} .
$$

Under the diffeomorphisms

$$
\delta_{\xi} A_{i}^{m} \equiv \xi^{j} F_{j i}^{m}-\xi^{0}\left(N^{j} F_{i j}^{m}+\Omega^{m n} \varepsilon_{i j k} \frac{\delta I}{\delta F_{j k}^{n}}\right),
$$

the full action $S$ transforms as

$$
\begin{aligned}
\delta_{\xi} S & =\int d^{4} x \xi^{0}\left(\frac{1}{4} \Omega_{m n} \partial_{1}\left(N^{2} h^{\mathrm{lh}} \varepsilon^{\mathrm{ijk}} F_{\mathrm{ih}}^{m} F_{j \mathrm{k}}^{n}\right)+\left(\partial_{0} F_{\mathrm{ij}}^{m}+2 \partial_{\mathrm{i}} N^{1} F_{j \mathrm{k}}^{m}\right) \frac{\delta I}{\delta F_{\mathrm{ij}}^{m}}\right)+\delta_{\xi} I \\
& =\int d^{4} x \xi^{0} \partial_{1}\left(\frac{1}{4} \Omega_{m n} N^{2} h^{\mathrm{lh}} \varepsilon^{\mathrm{ijk}} F_{\mathrm{ih}}^{m} F_{\mathrm{jk}}^{n}+\Omega^{m n} \varepsilon_{\mathrm{ijk}} \frac{\delta I}{\delta F_{\mathrm{il}}^{m}} \frac{\delta I}{\delta F_{\mathrm{jk}}^{n}}\right)+\bar{\delta}_{\xi} I
\end{aligned}
$$

where $\bar{\delta}_{\xi}$ is defined to act on $F_{i j}^{m}$ as an ordinary diffeomorphism according to (3.15)

$$
\bar{\delta}_{\xi} F_{\mathrm{ij}}^{m}=\xi^{\mu} \partial_{\mu} F_{\mu \nu}^{m}-2 F_{\mathrm{k}[\mathrm{i}} \partial_{\mathrm{j}]} \xi^{\mathrm{k}}+2\left(N^{\mathrm{k}} F_{[\mathrm{i} \mid \mathrm{k}}^{m}+\Omega^{m n} \varepsilon_{\mathrm{k} 1[\mathrm{i}} \frac{\delta I}{\delta F_{\mathrm{kl}}^{n}}\right) \partial_{\mathrm{j}]} \xi^{0} .
$$

The invariance of the action therefore follows from the vanishing of

$$
\frac{\delta^{L} \bar{\delta}_{\xi} I}{\delta \xi^{0}}=\partial_{1}\left(\frac{1}{4} \Omega_{m n} N^{2} h^{\mathrm{lh}} \varepsilon^{\mathrm{ijk}} F_{\mathrm{hi}}^{m} F_{\mathrm{jk}}^{n}+\Omega^{m n} \varepsilon_{\mathrm{ijk}} \frac{\delta I}{\delta F_{\mathrm{li}}^{m}} \frac{\delta I}{\delta F_{j \mathrm{k}}^{n}}\right) .
$$

This relation can be viewed as the non-covariant analogue of the consistency condition (1.8), but now ensuring space-time covariance of our manifestly duality invariant action.

The equation (3.19) defines a functional differential equation which permits to determine $I$ perturbatively. This equation simplifies drastically when $I$ contains no explicit

\footnotetext{
${ }^{7}$ The covariant derivative $\nabla_{\mu} F_{i j}^{m}$ must be defined perturbatively. At first order it is defined from the ordinary covariant derivative $\nabla_{\mu}$ acting on $F_{\mu \nu}^{m}$ as defined in (3.6),

$$
\nabla_{\mu}^{(0)} F_{i j}^{m}=\partial_{\mu} F_{i j}^{m}+2 \Gamma_{\mu[\mathbf{i}}^{\mathrm{k}} F_{j] \mathrm{k}}^{m}-2 \Gamma_{\mu[\mathbf{i}}^{0}\left(\partial_{0} A_{j]}^{m}-\mathscr{E}_{j]}^{m}\right)
$$
}


derivative terms, that is, when it is the integral of a polynomial function ('potential') $V$ of $F_{i j}^{m}$ and the metric, which is invariant under spatial diffeomorphisms. In that case (3.19) is no longer a functional differential equation, but reduces to the differential equation

$$
\frac{1}{4} \Omega_{m n} N^{2} h^{\mathrm{lh}} \varepsilon^{\mathrm{ijk}} F_{\mathrm{hi}}^{m} F_{\mathrm{jk}}^{n}=\Omega^{m n} \varepsilon_{\mathrm{ijk}} \frac{\delta I}{\delta F_{\mathrm{li}}^{m}} \frac{\delta I}{\delta F_{j \mathrm{k}}^{n}} \equiv \Omega^{m n} N^{2} h \varepsilon_{\mathrm{ijk}} \frac{\partial V}{\partial F_{\mathrm{li}}^{m}} \frac{\partial V}{\partial F_{j \mathrm{k}}^{n}} .
$$

\section{Maxwell theory, once again}

To illustrate how the procedure works in the non-covariant formulation we again study an example generalising Maxwell theory. To keep things as simple as possible we consider a modification that initially depends on the complex spatial field strength $F_{\text {ij }}$ polynomially, but not on its derivatives (the inclusion of derivatives presents no problem of principle, but renders the calculations substantially more tedious). The tree level Lagrangian is now a function of the complex spatial vector field $A_{\mathrm{i}}=A_{\mathrm{i}}^{1}+i A_{\mathrm{i}}^{2}$ and reads

$$
\mathcal{L}=-\frac{i}{2} \varepsilon^{\mathrm{ijk}}\left(\partial_{0} A_{\mathrm{i}}+N^{\mathrm{l}} F_{\mathrm{il}}\right) \bar{F}_{\mathrm{jk}}+\frac{i}{2} \varepsilon^{\mathrm{ijk}}\left(\partial_{0} \bar{A}_{\mathrm{i}}+N^{\mathrm{l}} \bar{F}_{\mathrm{il}}\right) F_{\mathrm{jk}}-N \sqrt{h} V[F] .
$$

with the tree level 'potential'

$$
V \equiv V^{(0)}=h^{\mathrm{ik}} h^{\mathrm{jl}} F_{\mathrm{ij}} \bar{F}_{\mathrm{kl}}=F^{a b} \bar{F}_{a b}
$$

Here and in the remainder, we will mostly use flat indices

$$
F_{a b} \equiv e_{a}^{i} e_{b}^{j} F_{\mathrm{ij}},
$$

where $e_{a}^{\mathrm{i}}$ is the inverse dreibein such that $h^{\mathrm{ij}}=\delta^{a b} e_{a}^{\mathrm{i}} e_{b}^{\mathrm{j}}$. Generalising beyond tree level, the potential $V$ will be a more complicated function, but for any given $V$, the three vector transforms as

$$
\delta A_{\mathrm{i}}=\left(\xi^{\mathrm{j}}+\xi^{0} N^{\mathrm{j}}\right) F_{\mathrm{ji}}-i \xi^{0} \frac{N \sqrt{h}}{2} \varepsilon_{\mathrm{ijk}} \frac{\partial V}{\partial \bar{F}_{\mathrm{jk}}},
$$

In order to ensure full diffeomorphism invariance, $V$ must satisfy the consistency condition (3.19) which now reads

$$
\frac{\partial V}{\partial F^{a[b}} \frac{\partial V}{\left.\partial \bar{F}^{c d}\right]}=\bar{F}_{a[b} F_{c d]} .
$$

The general procedure then starts from some 'initial' corrected potential of the form $V=V^{(0)}+V^{(1)}$ and exploits (4.5) in order to complete the potential $V$ to a more general $\mathrm{SO}(3)$ invariant function of the spatial field strengths $F_{a b}$ and $\bar{F}_{a b}$, such that

$$
V=V^{(0)}+V^{(1)}+\cdots
$$

satisfies the differential equation (3.19). As before we will thus find that, for consistency, any 'initial' counterterm $V^{(1)}$ must be supplemented by an infinite string of higher order corrections. As the simpest possible example we will consider the manifestly duality invariant expression $V^{(1)} \propto \frac{1}{2} F_{\alpha \beta} F^{\alpha \beta} F_{\dot{\alpha} \dot{\beta}} F^{\dot{\alpha} \dot{\beta}}$ obtained by squaring the complex selfdual and 
anti-selfdual field strengths. In the present approach this invariant can be identified with one half the duality invariant

$$
Y \equiv F^{a b} F_{a b} \bar{F}^{c d} \bar{F}_{c d}
$$

by using the equations of motion. Writing also

$$
X \equiv F^{a b} \bar{F}_{a b},
$$

we would thus like to solve (4.5) for

$$
V(X, Y)=F^{a b} \bar{F}_{a b}+\frac{1}{2} F^{a b} F_{a b} \bar{F}^{c d} \bar{F}_{c d}+\mathcal{O}\left(F^{6}\right) \equiv X+\frac{1}{2} Y+\mathcal{O}\left(F^{6}\right)
$$

First of all we note that (4.5) is trivially satisfied at first order because

$$
F_{a[b} F_{c d]}=0 .
$$

After some further computation it is seen that $V$ must be of the form

$$
V(X, Y)=X+\sum_{n=0}^{\infty} \frac{1}{(2+2 n) !} H^{(n)}(X) Y^{1+n}
$$

The condition (4.5) is satisfied provided (using $\bar{F}_{a[b} F_{c d]}=-F_{a[b} \bar{F}_{c d]}$ )

$$
\left(1+\sum_{n=0}^{\infty} \frac{Y^{1+n}}{(2+2 n) !} \frac{\partial H^{(n)}}{\partial X}\right)^{2}=Y\left(\sum_{n=0}^{\infty} \frac{Y^{n}}{(1+2 n) !} H^{(n)}\right)^{2}+1 .
$$

Observe that this ansatz is manifestly duality invariant. At first order in $Y$ one gets

$$
\frac{\partial H^{(0)}}{\partial X}=\left(H^{(0)}\right)^{2}
$$

which implies (with the condition $H^{(0)}(0)=1$ ) that

$$
H^{(0)}(X)=\frac{1}{1-X} \text {. }
$$

At order $Y^{2}$ we get

$$
\frac{\partial H^{(1)}}{\partial X}-\frac{4}{1-X} H^{(1)}=-\frac{3}{(1-X)^{2}}
$$

which gives

$$
H^{(1)}(X)=\frac{1}{1-X}+\frac{c^{(1)}}{(1-X)^{4}},
$$

with an arbitrary constant $c^{(1)}$. This constant corresponds to the freedom of adding the on-shell invariant

$$
\frac{c^{(1)}}{24}\left(F_{a b} F_{a b} \bar{F}_{c d} \bar{F}_{c d}\right)^{2} \approx \frac{c^{(1)}}{24}\left(F_{\alpha \beta} F^{\alpha \beta} F_{\dot{\alpha} \dot{\beta}} F^{\dot{\alpha} \dot{\beta}}\right)^{2}
$$

to the invariant $F^{2} \bar{F}^{2}$, while preserving diffeomorphism invariance. 
It is now clear how to proceed perturbatively in $Y$ and how to determine all the functions $H^{(n)}$ by successively solving the hierarchy of first order equations ${ }^{8}$

$$
\begin{aligned}
& \frac{\partial}{\partial X}\left((1-X)^{2+2 n} H^{(n)}\right)= \\
& \quad \frac{1}{2}(1-X)^{2+2 n}\left(\sum_{p=1}^{n-1} C_{2+2 n}^{1+2 p} H^{(p)} H^{(n-p)}-\sum_{p=0}^{n-1} C_{2+2 n}^{2+2 p} \frac{\partial H^{(p)}}{\partial X} \frac{\partial H^{(n-p-1)}}{\partial X}\right) .
\end{aligned}
$$

By construction the right-hand side is a finite Laurent series in $(1-X)$ with polynomial coefficients in $\ln (1-X)$ which can be integrated straightforwardly, modulo the definition of the homogenous solution

$$
H^{(n)}=\frac{c^{(n)}}{(1-X)^{2+2 n}}+\tilde{H}^{(n)}
$$

$\tilde{H}^{(n)}$ being a particular solution. Clearly, the constants $c^{(n)}$ correspond to the ambiguities in defining a diffeormorphism invariant associated to the possibility of adding higher order invariants corresponding on-shell to

$$
\frac{c^{(n)}}{(2 n) !}\left(F_{\alpha \beta} F^{\alpha \beta}\right)^{n}\left(F_{\dot{\alpha} \dot{\beta}} F^{\dot{\alpha} \dot{\beta}}\right)^{n} \approx \frac{c^{(n)}}{(2 n) !}\left(F_{a b} F_{a b}\right)^{n}\left(\bar{F}_{c d} \bar{F}_{c d}\right)^{n} .
$$

Setting $c^{(1)}=5$, the potential $V$ reads

$$
V(X, Y)=X+\frac{1}{2} Y\left(1+X+X^{2}\right)+\frac{1}{4} Y^{2}+\mathcal{O}\left(F^{10}\right) .
$$

To establish the link of the above construction with the deformed twisted self-duality constraint (2.1), we note that, by construction, the equations of motion are invariant with respect to diffeomorphism invariance. Hence they can indeed be rewritten in this manifestly diffeomorphism covariant form (for $c^{(1)}=5$ and higher $c^{(n)}$ chosen appropriately), viz.

$$
F_{\mu \nu}-\frac{i}{2 \sqrt{-g}} \varepsilon_{\mu \nu}^{\sigma \rho} F_{\sigma \rho}+\frac{1}{8}\left(F_{\kappa \lambda} F^{\kappa \lambda}+\frac{i}{2 \sqrt{-g}} \varepsilon^{\kappa \lambda \theta \tau} F_{\kappa \lambda} F_{\theta \tau}\right)\left(\bar{F}_{\mu \nu}-\frac{i}{2 \sqrt{-g}} \varepsilon_{\mu \nu}{ }^{\sigma \rho} \bar{F}_{\sigma \rho}\right)=0 .
$$

Indeed, decomposing the corresponding equations into space and time components

$$
F_{0 a}-\frac{i}{2} \varepsilon_{a b c} F_{b c}+\frac{1}{8}\left(F_{d e} F_{d e}-2 F_{o d} F_{o d}-2 i \varepsilon_{d e f} F_{0 d} F_{e f}\right)\left(\bar{F}_{0 a}-\frac{i}{2} \varepsilon_{a b c} \bar{F}_{b c}\right)=0,
$$

one can perturbatively solve for $F_{0 a}$ in terms of $F_{a b}$ as

$$
\begin{aligned}
F_{0 a}=\frac{i}{2} \varepsilon_{a b c} F_{b c}\left(1+\frac{1}{2} Y(1+2 X)\right) & \\
& +\frac{i}{2} \varepsilon_{a}{ }^{b c} \bar{F}_{b c} F_{e f} F_{e f}\left(1+X+X^{2}+Y\right)+\mathcal{O}\left(F^{10}\right),
\end{aligned}
$$

This solution coincides with the expression following from the corrected potential $V$ obtained above in (4.21) up to order $F^{10}$.

\footnotetext{
${ }^{8}$ Where $C_{n}^{p} \equiv \frac{n !}{p !(n-p) !}$ are the binomial coefficients.
} 


\section{Conclusions}

We have demonstrated for some typical examples by rather explicit computations that the higher order counterterms and corrections arising in supergravity and the effective string theory action are perfectly compatible with the full non-linear duality symmetries of these theories, provided one completes the 'initial' correction terms by solving the requisite consistency conditions. This can be done in either of two different formulations, in one of which space-time covariance is manifest but the duality symmetry is realised only onshell, while it is the converse in the second formulation. We have exhibited the analogue of the Gaillard-Zumino constraint for the Henneaux-Teitelboim formulation, and we have furthermore shown that the two procedures give results which agree at lowest non-trivial orders in a perturbative expansion.

We have shown that the absence of diffeormorphism anomalies in four dimensions implies that one can always construct a diffeomorphism invariant corrected action functional associated to an 'on-shell' invariant in the Henneaux-Teitelboim formulation. We argued that the absence of supersymmetry anomalies similarly implies that one can always construct a supersymmetric corrected action functional associated to any given 'on-shell' invariant.

We conclude that the non-linear $E_{7(7)}$ symmetry is not sufficient to rule out all higher order counterterms, hence divergences, of $\mathcal{N}=8$ supergravity. At this stage of our understanding of the theory, there is unfortunately no 'royal path' to finiteness cutting short explicit calculations of the type performed in [20]. If $\mathcal{N}=8$ supergravity is UV finite to all orders the reason must be sought beyond maximal supersymmetry and $E_{7(7)}$.

\section{Acknowledgments}

H. Nicolai would like to thank the Simons Center at Stony Brook and its staff for hospitality and support during part of this work. G. Bossard is grateful to AEI Potsdam for hospitality. We would furthermore like to thank R. Kallosh for discussions and comments motivating this work.

\section{A Deforming the NGZ identity}

In this appendix we discuss the NGZ identities required for the invariance of the equations of motion under duality [2-4] and their deformation when the original action is deformed by higher order corrections. For ease of comparison we adopt the notation and conventions of [4] throughout this appendix. Suppressing the dependence on other fields as well as all indices for simplicity, we write the action as $S=S[F]$, where, as everywhere else in this paper, the term 'action' always refers to a functional depending on $n$ electric vectors and their associated field strengths $F$ only. We then define the magnetic field strengths via

$$
\tilde{G}[F]:=2 \frac{\delta S[F]}{\delta F}
$$


as functionals of $F$ in the standard way. If the equations of motion are to be duality covariant under

$$
\Delta F=A F+B G \quad, \quad \Delta G=C F+D G
$$

with the usual $\operatorname{Sp}(2 n, \mathbb{R})$ matrix relations $A^{T}=-D, B=B^{T}$ and $C=C^{T}$, the action must satisfy the NGZ constraint [2] (三 eq. (3.6) in [4]))

$$
\frac{\delta}{\delta F}\left(S\left[F^{\prime}\right]-S[F]-\frac{1}{4} \int(\tilde{F} C F+\tilde{G} B G)\right)=0
$$

This is necessary for the compatibility of the transformations (A.2) with the constitutive relations (A.1), which imply

$$
\Delta G[F]=\int \frac{\delta G}{\delta F} \Delta F
$$

Now assume that these conditions are satisfied for some initial (usually the tree level) action $S_{0}[F]$ with corresponding

$$
\tilde{G}_{0}[F]:=2 \frac{\delta S_{0}[F]}{\delta F}
$$

We wish to 'deform' this action and investigate how the duality symmetry is deformed with it. To this aim we expand the full action $S$ as

$$
S[F ; \lambda]=S_{0}[F]+\lambda S_{1}[F]+\mathcal{O}\left(\lambda^{2}\right)
$$

with some expansion parameter $\lambda$. Similarly,

$$
\tilde{G}[F ; \lambda]:=2 \frac{\delta S[F ; \lambda]}{\delta F}=\tilde{G}_{0}[F]+\lambda \tilde{G}_{1}[F]+\mathcal{O}\left(\lambda^{2}\right)
$$

such that

$$
\tilde{G}_{k}[F]:=2 \frac{\delta S_{k}[F]}{\delta F}
$$

for the $k$-th order correction to the magnetic field strengths. The expansion of the variation is

$$
\Delta(\lambda)=\Delta_{0}+\lambda \Delta_{1}+\mathcal{O}\left(\lambda^{2}\right)
$$

Inserting this into (A.2) we get

$$
\left(\Delta_{0}+\lambda \Delta_{1}+\ldots\right) F=A F+B\left(G_{0}+\lambda G_{1}+\ldots\right)
$$

or

$$
\Delta_{0} F=A F+B G_{0}, \quad \Delta_{k} F=B G_{k}
$$

and

$$
\left(\Delta_{0}+\lambda \Delta_{1}+\ldots\right)\left(G_{0}+\lambda G_{1}+\ldots\right)=C F+D\left(G_{0}+\lambda G_{1}+\ldots\right)
$$

Similarly we can expand the NGZ relation (A.3)

$$
\begin{array}{r}
\frac{\delta}{\delta F}\left\{S_{0}[F+\Delta F]+\lambda S_{1}[F+\Delta F]-S_{0}[F]-\lambda S_{1}[F]-\cdots-\frac{1}{4} \int \tilde{F} C F\right. \\
\left.-\frac{1}{4} \int\left(\tilde{G}_{0}+\lambda \tilde{G}_{1}+\ldots\right) B\left(G_{0}+\lambda G_{1}+\ldots\right)\right\}=0
\end{array}
$$


By assumption this relation holds at 0 -th order in $\lambda$. At first order in $\lambda$ we get

$$
\int\left(\frac{\delta S_{0}}{\delta F} \Delta_{1} F+\frac{\delta S_{1}}{\delta F} \Delta_{0} F-\frac{1}{2} \tilde{G}_{0} B G_{1}\right)=\int \frac{\delta S_{1}[F]}{\delta F} \Delta_{0} F \equiv \Delta_{0} S_{1}
$$

where we used (A.5) and (A.11). Therefore, to this order duality can be maintained if and only if the first order correction to the action is invariant under undeformed duality, or

$$
\frac{\delta}{\delta F}\left(\Delta_{0} S_{1}[F]\right)=0 \Rightarrow \Delta_{0} S_{1}[F]=0 .
$$

This can be arranged by starting from a manifestly duality invariant functional $\mathcal{I}=\mathcal{I}[F, G]$ obeying

$$
\Delta \mathcal{I}=\int\left(\frac{\delta \mathcal{I}}{\delta F}(A F+B G)+\frac{\delta \mathcal{I}}{\delta G}(C F+D G)\right)=0
$$

for arbitrary (hence independent) $F$ and $G$, but in particular also for the special choice $G=G_{0}[F]$ and $\Delta=\Delta_{0}$. If we now take

$$
S_{1}[F]:=\mathcal{I}\left[F, G_{0}[F]\right]
$$

the 0 -th order variation comes from the varying $F$ alone

$$
\Delta_{0} S_{1}[F]=\int\left(\frac{\delta \mathcal{I}\left[F, G_{0}(F)\right]}{\delta F}+\frac{\delta \mathcal{I}\left[F, G_{0}(F)\right]}{\delta G_{0}} \frac{\delta G_{0}}{\delta F}\right) \Delta_{0} F
$$

Using (A.4) together with (A.15) we see that indeed $\Delta_{0} S_{1}=0$ as required, and duality is maintained at $\mathcal{O}(\lambda)$. Therefore, the first order correction is always invariant under the undeformed duality, as borne out also by the examples from $\mathcal{N}=8$ supergravity.

Up to this point our analysis agrees with [4] . At second order, however, we disagree with the conclusions of [4]. Collecting all terms of order $\mathcal{O}\left(\lambda^{2}\right)$ in (A.13) we obtain

$$
\begin{aligned}
\frac{\delta}{\delta F}\left\{\int\right. & \left.\left(\frac{\delta S_{0}}{\delta F} \Delta_{2} F+\frac{\delta S_{1}}{\delta F} \Delta_{1} F+\frac{\delta S_{2}}{\delta F} \Delta_{0} F-\frac{1}{4} \tilde{G}_{1} B G_{1}-\frac{1}{2} \tilde{G}_{2} B G_{0}\right)\right\} \\
= & \frac{\delta}{\delta F}\left\{\int\left(\frac{\delta S_{2}}{\delta F} \Delta_{0} F+\frac{1}{4} \tilde{G}_{1} B G_{1}\right)\right\} \\
& =0
\end{aligned}
$$

(we drop all terms of higher order in any of the $\Delta$ 's because the matrices $A, B, C, D$ are assumed to be infinitesimal). In this derivation we made use again of (A.8) and (A.11) in order to have only one unknown quantity $\left(=S_{2}\right)$ in this expression. It is now clear that we must include a second order term $S_{2} \neq 0$ (and correspondingly higher order corrections) to salvage duality invariance at this order (and higher orders). Furthermore, unlike the first order correction, $S_{2}$ cannot be invariant under undeformed duality, but must break undeformed duality in such a way as to cancel the second term on the r.h.s. of (A.18); that is, we must have $\Delta_{0} S_{2} \neq 0$. This also implies

$$
\Delta_{0}\left(\lambda S_{1}+\lambda^{2} S_{2}+\ldots\right)=\mathcal{O}\left(\lambda^{2}\right) \neq 0
$$


so (3.9) of [4] (stating $\Delta_{0} \hat{S}=0$ ) is only correct to first order in $\lambda$, but fails beyond. Let us finally note that, assuming integrability of the resulting functional differential equations, the expansion (A.6) offers an alternative method for constructing the deformed duality invariant action order by order as a formal power series.

Open Access. This article is distributed under the terms of the Creative Commons Attribution Noncommercial License which permits any noncommercial use, distribution, and reproduction in any medium, provided the original author(s) and source are credited.

\section{References}

[1] E. Cremmer and B. Julia, The SO(8) supergravity, Nucl. Phys. B 159 (1979) 141 [SPIRES].

[2] M.K. Gaillard and B. Zumino, Duality rotations for interacting fields, Nucl. Phys. B 193 (1981) 221 [SPIRES].

[3] P. Aschieri, S. Ferrara and B. Zumino, Duality rotations in nonlinear electrodynamics and in extended supergravity, Riv. Nuovo Cim. 31 (2008) 625 [arXiv:0807.4039] [SPIRES].

[4] R. Kallosh, $E_{7(7)}$ symmetry and finiteness of $N=8$ supergravity, arXiv:1103.4115 [SPIRES].

[5] G. Bossard, C. Hillmann and H. Nicolai, $E_{7(7)}$ symmetry in perturbatively quantised $N=8$ supergravity, JHEP 12 (2010) 052 [arXiv:1007.5472] [SPIRES].

[6] C. Hillmann, $E_{7(7)}$ invariant lagrangian of $D=4 N=8$ supergravity, JHEP 04 (2010) 010 [arXiv:0911.5225] [SPIRES].

[7] P.S. Howe and U. Lindström, Higher order invariants in extended supergravity, Nucl. Phys. B 181 (1981) 487 [SPIRES].

[8] R.E. Kallosh, Counterterms in extended supergravities, Phys. Lett. B 99 (1981) 122 [SPIRES].

[9] P.S. Howe, K.S. Stelle and P.K. Townsend, Superactions, Nucl. Phys. B 191 (1981) 445 [SPIRES].

[10] R. Kallosh, $N=8$ counterterms and $E_{7(7)}$ current conservation, JHEP 06 (2011) 073 [arXiv:1104.5480] [SPIRES].

[11] D.Z. Freedman and E. Tonni, The $D^{2 k} R^{4}$ invariants of $N=8$ supergravity, JHEP 04 (2011) 006 [arXiv:1101.1672] [SPIRES].

[12] H. Elvang and M. Kiermaier, Stringy KLT relations, global symmetries and $E_{7(7)}$ violation, JHEP 10 (2010) 108 [arXiv: 1007.4813] [SPIRES].

[13] G. Bossard, P.S. Howe and K.S. Stelle, On duality symmetries of supergravity invariants, JHEP 01 (2011) 020 [arXiv: 1009.0743] [SPIRES].

[14] M.B. Green, J.G. Russo and P. Vanhove, Automorphic properties of low energy string amplitudes in various dimensions, Phys. Rev. D 81 (2010) 086008 [arXiv:1001.2535] [SPIRES].

[15] B. de Wit and H. Nicolai, $N=8$ supergravity, Nucl. Phys. B 208 (1982) 323 [SPIRES].

[16] M. Henneaux and C. Teitelboim, Dynamics of chiral (selfdual) p-forms, Phys. Lett. B 206 (1988) 650 [SPIRES]. 
[17] G. Barnich, F. Brandt and M. Henneaux, Local BRST cohomology in the antifield formalism. 1. General theorems, Commun. Math. Phys. 174 (1995) 57 [hep-th/9405109] [SPIRES].

[18] L. Álvarez-Gaumé and E. Witten, Gravitational anomalies, Nucl. Phys. B 234 (1984) 269 [SPIRES].

[19] O. Alvarez, I.M. Singer and B. Zumino, Gravitational anomalies and the family's index theorem, Commun. Math. Phys. 96 (1984) 409 [SPIRES].

[20] Z. Bern, J.J. Carrasco, L.J. Dixon, H. Johansson and R. Roiban, The ultraviolet behavior of $N=8$ supergravity at four loops, Phys. Rev. Lett. 103 (2009) 081301 [arXiv:0905.2326] [SPIRES]. 